\title{
ONCOGENOMICS
}

\section{Using a novel computational drug-repositioning approach (DrugPredict) to rapidly identify potent drug candidates} for cancer treatment

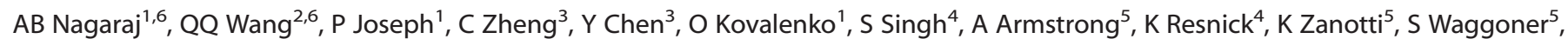

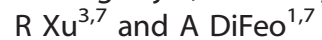

Computation-based drug-repurposing/repositioning approaches can greatly speed up the traditional drug discovery process. To date, systematic and comprehensive computation-based approaches to identify and validate drug-repositioning candidates for epithelial ovarian cancer (EOC) have not been undertaken. Here, we present a novel drug discovery strategy that combines a computational drug-repositioning system (DrugPredict) with biological testing in cell lines in order to rapidly identify novel drug candidates for EOC. DrugPredict exploited unique repositioning opportunities rendered by a vast amount of disease genomics, phenomics, drug treatment, and genetic pathway and uniquely revealed that non-steroidal anti-inflammatories (NSAIDs) rank just as high as currently used ovarian cancer drugs. As epidemiological studies have reported decreased incidence of ovarian cancer associated with regular intake of NSAIDs, we assessed whether NSAIDs could have chemoadjuvant applications in EOC and found that (i) NSAID Indomethacin induces robust cell death in primary patient-derived platinum-sensitive and platinum- resistant ovarian cancer cells and ovarian cancer stem cells and (ii) downregulation of $\beta$-catenin is partially driving effects of Indomethacin in cisplatin-resistant cells. In summary, we demonstrate that DrugPredict represents an innovative computational drug- discovery strategy to uncover drugs that are routinely used for other indications that could be effective in treating various cancers, thus introducing a potentially rapid and cost-effective translational opportunity. As NSAIDs are already in routine use in gynecological treatment regimens and have acceptable safety profile, our results will provide with a rationale for testing NSAIDs as potential chemoadjuvants in EOC patient trials.

Oncogene (2018) 37, 403-414; doi:10.1038/onc.2017.328; published online 2 October 2017

\section{INTRODUCTION}

Epithelial ovarian cancer (EOC) is the most lethal gynecologic malignancy and is the fifth leading cause of cancer deaths in women. ${ }^{1}$ This year it is estimated that $\sim 22000$ women will be newly diagnosed and $>14000$ will succumb to this lethal disease in the United States. ${ }^{1}$ Most cases of EOC are high-grade serous cancers HGSOC, which are initially highly sensitive to standard treatment of cytoreduction surgery and platinum-based chemotherapy with response rate close to $85 \% .{ }^{2}$ In spite of such high initial response to platinum the outcomes are poor with 5-year survival being $<30 \%$ and the majority of women who initially respond to platinum therapy relapse due to development of chemoresistance. ${ }^{2}$ Therefore, novel therapeutic options are required in order to improve the lives of women diagnosed with HGSOC.

Traditional drug development is expensive and time-consuming, taking an average of 14 years and costing $>\$ 2$ billion to bring a drug to market. During drug development, $~ 90 \%$ of drugs fail because of safety concerns or lack of efficacy. 3,4
Computation-based drug-repositioning approaches that automatically integrate and analyze vast amounts of data for tens of thousands of drugs and diseases can greatly speed up the traditional drug discovery process. ${ }^{5}$ The primary advantage of drug repositioning is that it starts from compounds with wellcharacterized pharmacology and safety profiles that can greatly reduce the risk of attrition in drug development in clinical phases. ${ }^{6}$ We have recently developed novel computational algorithms that identified repurposed drug candidates to treat neuropsychiatric disorders, including schizophrenia ${ }^{7}$ and Parkinson's disease, ${ }^{8-10}$ infectious diseases including dengue fever ${ }^{11}$ and malaria; ${ }^{12}$ cancers including glioblastoma; ${ }^{13,14}$ and immune-mediated diseases including Crohn's disease, ${ }^{15}$ inflammatory bowel disease ${ }^{16}$ and rheumatoid arthritis. ${ }^{17}$ However, to date, systematic and comprehensive computation-based approaches to identify and validate drug-repositioning candidates for HGSOC have not been undertaken. Here we present a novel drug discovery strategy that combines a novel computational drug-repositioning system (DrugPredict) and experimental validation to rapidly identify

\footnotetext{
${ }^{1}$ Case Comprehensive Cancer Center, Case Western Reserve University, Cleveland, OH, USA; ${ }^{2}$ ThinTek LLC, Palo Alto, CA, USA; ${ }^{3}$ The Department of Population and Quantitative Health Sciences, Institute of Computational Biology, Case Western Reserve University, Cleveland, OH, USA; ${ }^{4}$ Department of Gynecology, Division of Gynecological Oncology, MetroHealth Medical Center, Cleveland, $\mathrm{OH}$, USA and ${ }^{5}$ Department of Gynecology, Division of Gynecological Oncology, University Hospital Cleveland Medical Center, Cleveland, $\mathrm{OH}$, USA. Correspondence: Dr R Xu, Department of Epidemiology and Biostatistics, Institute of Computational Biology, School of Medicine, Case Western Reserve University, 2103 Cornell Road, Cleveland, OH 44106, USA or Dr A DiFeo, General Medical Sciences (Oncology), Case Comprehensive Cancer Center, 2103 Cornell Road, Cleveland, Ohio 44106, USA. E-mail: rxx@case.edu or avd11@case.edu

${ }^{6}$ These authors contributed equally to this work.

${ }^{7}$ These authors contributed equally to this work.

Received 2 February 2017; revised 28 June 2017; accepted 3 July 2017; published online 2 October 2017
} 
repositioned drug candidates for the treatment of HGSOC. Interestingly, among top-ranked drug candidates predicted by DrugPredict, two COX-2 selective inhibitors (Celecoxib, Nimesulide) and one non-selective COX inhibitor (Indomethacin) were in the top three ranked non-steroidal anti-inflammatories (NSAIDs), higher than top 3.5\% ranking for carboplatin which is used as standard therapy for the majority of EOC patients. Intriguingly, several epidemiological studies have suggested that regular intake of NSAIDs in women could reduce the risk of ovarian cancer. ${ }^{18-21}$

Indomethacin (ranked at top $0.5 \%$ ) is one of the most potent NSAIDs and is a common constituent of rheumatic disease treatment regimens in women to decrease the inflammationassociated pathogenesis. However, the use of NSAIDs as chemoadjuvant compounds is not very well understood in ovarian cancer. In this study, we found that Indomethacin inhibits cellular survival and induces apoptosis in primary HGSOC patient-derived cell lines with minimal effect on non-transformed fallopian tube and ovarian surface epithelium. Functionally, we show that Indomethacin inhibits $\beta$-catenin and represses several Wnt signaling targets including Lgr5 and Axin2. In addition, we found that platinum-resistant ovarian tumor cells were more sensitive to Indomethacin potentially due to the dependency on $\beta$-catenin. Together, through the combination of a novel computational drug-repositioning system and functional validation of a highly ranked drug candidate we uncovered that NSAIDs, namely Indomethacin, may have potential chemoadjuvant applications in HGSOC.

\section{RESULTS}

DrugPredict: a computational drug-repurposing system

Current repositioning strategies can be categorized as drug-based, disease-based and profile-based. Drug-based and disease-based approaches exploit drug-drug or disease-disease similarity and existing drug-treatment knowledge to infer new disease-drug associations. $^{22,23}$ Drug similarities are often based on pharmacological data, such as drug chemical structure and drug side-effects. Disease similarities are often calculated using disease phenotype, disease genetic and genomic data. Recently, profile-based repositioning strategies have successfully found new drug candidates for inflammatory bowel disease $\mathrm{e}^{24}$ and small cell lung cancer. ${ }^{25}$ Here we present DrugPredict, which is a profile-based drug-repositioning system. Compared with both drug- and disease-based approaches, profile-based approaches do not rely on the knowledge of existing drug treatments, and have increased ability to discover new drug-disease pairs compared with drugbased and disease-based strategies. Existing profile-based drugrepositioning approaches mainly exploit the gene expression profiles of drugs and diseases, and have an inherent challenge: the lower-level genomics profile similarities between drugs and diseases do not necessarily translate into higher-level drugtreatment efficacy in diseases. Recently, the MGI (Mouse Genome Informatics) has made available large amounts of phenotypic descriptions for mouse genetic mutations based on systematic gene knockouts, ${ }^{26}$ which are impossible on human. These causal gene-phenotype associations in mice have been demonstrated useful in discovering new drug targets ${ }^{27}$ and also have the potential to overcome the challenge in profile-based drugrepositioning approaches.

DrugPredict is a profile-based drug-repositioning system that performs both genome- and phenome-wide analysis to match disease to drug candidates. For a given disease (EOC in this study), DrugPredict first finds the mouse mutational phenotype profile that is significantly enriched for the disease-associated genes. It then systematically scans all chemicals/drugs to identify/prioritize them based on the similarities between disease-specific phenotype profile and drug-specific phenotype profiles. The inputs to
DrugPredict are a specific disease or a set of disease-associated genes, mouse genome mutation phenotype data, chemical/drug genetics. The output is a list of drugs ranked based on how likely the drugs can be used to treat the disease.

The experimental outline in applying DrugPredict for OC drug repurposing is shown in Figure 1 and consists of the following steps: (1) obtain a list of genes that are differentially expressed in high-grade serous ovarian cancer (HGSOC) patients from TCGA (HGSOC genes); (2) correlate HGSOC genes to their homologs in mouse models and construct a mouse mutational phenotype profile for HGSOC genes; (2) construct mouse mutational phenotype profile for chemicals/drugs; (3) develop algorithms to match the HGSOC-specific phenotype profile to drug profiles and prioritize Food and Drug Administration (FDA)-approved drugs based on the phenotypic similarities; and (4) analyze repositioned drug candidates to evaluate the predictions and identify interpretable mechanisms of actions.

\section{DrugPredict ranked FDA-approved EOC drugs highly}

We first evaluated the performance of DrugPredict using 16 FDAapproved EOC drugs. DrugPredict found 15 FDA-approved EOC drugs (recall $=0.94$ ) and ranked them highly. Among a total of 6996 prioritized chemicals (including 1096 FDA-approved drugs), the 15 FDA-approved EOC drugs had a median ranking of $1.34 \%$ and mean ranking of $6.44 \%$ (Figure $2 a$ ). For example, paclitaxel, carboplatin and cisplatin that are used as first-line standard therapy ranked among the top 10\%: paclitaxel ranked at $0.1 \%$, carboplatin at $3.7 \%$ and cisplatin at $4.5 \%$ (Figure 2a).

\section{NSAID ranked significantly high}

Among the 6996 prioritized chemicals, a total of 1096 are FDAapproved drugs. We classified these, 1096 drugs using the ATC (Anatomical Therapeutic Chemical) Classification System codes. Among a total of 882 fourth-level drug classes, 31 classes of drugs ranked significantly higher than random expectation. As expected, anticancer drugs (platinum compounds, pyrimidine analogs and other antineoplastic agents) ranked highest (Figure 2b). For example, the pyrimidine analogs (for example, azacitidine, gemcitabine, capecitabine, fluorouracil) have a mean ranking of $2.26 \%$ and a median ranking of $2.09 \%$, which rank significantly higher than random $(P<1.74 \mathrm{E}-08)$ : azacitidine ranked at $0.34 \%$, gemcitabine at $0.80 \%$, capecitabine at $3.33 \%$ and fluorouracil at $3.49 \%$. The fact that anticancer drugs ranked highly demonstrates the validity of DrugPredict. Interestingly, the drug class 'Antiinflammatory preparations, non-steroid' was also ranked significantly high $(P<0.009)$, with a mean ranking of $23.55 \%$ and a median ranking of $14.08 \%$ (Figure $2 \mathrm{~b}$ ). The top three ranked NSAIDs were 2 COX-2 selective inhibitors (Celecoxib, Nimesulide) and one non-selective COX inhibitor (Indomethacin). Nimesulide is a controversial drug and is under limited use in some countries and hence we did not include it in our study. ${ }^{28}$ Celecoxib is known to be effective against ovarian cancer cells but it is reported to induce epithelial-mesenchymal transition in ovarian cancer thus raising concerns about using it as a potential therapeutic option in ovarian cancer. ${ }^{29}$ In addition, COX-2 selective inhibitors like Celecoxib are known to increase risk of thromboembolism and a recent meta-analysis of $>21000$ cases of venous thromboembolism (VTE) showed an increased risk of VTE with use of COX-2 selective inhibitors. ${ }^{30,31}$ VTE is an important determinant while considering novel therapeutic options in ovarian cancer, as VTE incidence is very high in ovarian cancer compared with other cancers and in fact this is further complicated by the fact that cisplatin chemotherapy is associated with a high incidence of thromboembolic complications. ${ }^{32,33}$ Hence, we focused on the non-selective COX inhibitor Indomethacin that is on the contrary shown to be a possible treatment option to decrease VTE complications ${ }^{34}$ and its role as a chemoadjuvant in ovarian cancer 
HGSOC TCGA dataset

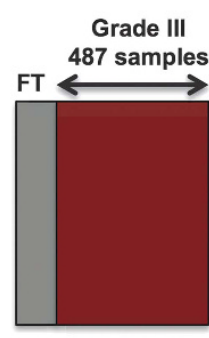

HGSOC specific phenotype profile

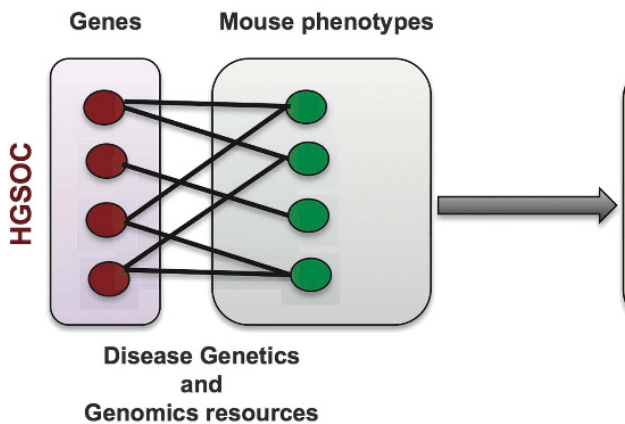

Prioritize drugs

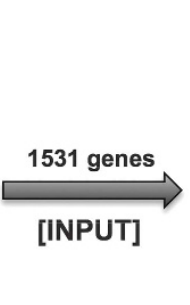

Genomics resources
Analysis in HGSOC PDX models OV81 PDX

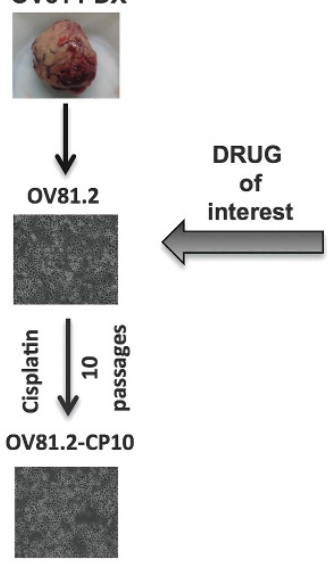

Top ranked drug classes

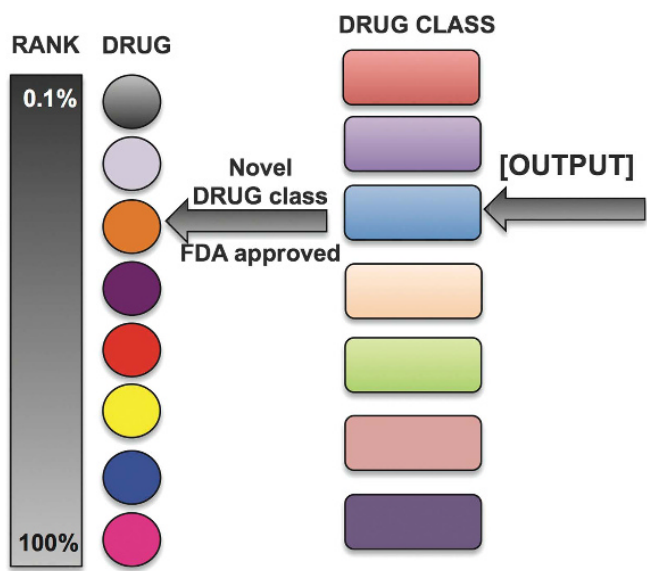

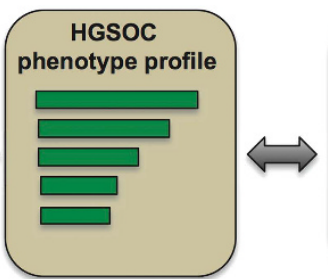

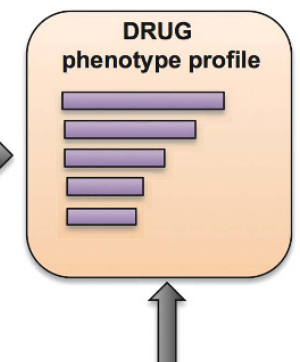

DRUG specific phenotype profile

Figure 1. Schematic showing outline of DrugPredict approach.

has yet to be explored. Indomethacin is also reported to be effective in eradicating tumor-initiating cells (TICs) in cancers ${ }^{35}$ and these TICs are identified to be the root cause of chemoresistance and tumor recurrence in ovarian cancer. ${ }^{36}$ Hence, Indomethacin could be evaluated as a potential TIC targeting drug in ovarian cancer. Also, an ongoing phase I trial is currently evaluating the efficacy of combining Indomethacin with Cisplatin in ovarian cancer patients (NCT01719926), which further prompted us to focus on evaluating Indomethacin as a potential chemoadjuvant in ovarian cancer.

Indomethacin decreases survival of both platinum-sensitive and platinum-resistant ovarian tumor cells

We first looked at the effect of Indomethacin on primary HGSOC ovarian tumor cells. Indomethacin decreased cellular viability in all primary serous ovarian tumor cells tested (OV78: Stage IV HGSOC, OV81: Stage IIIC HGSOC, OV82: Stage IIIC low-grade serous EOC, OV84: Stage IIIC HGSOC) (Figure 3a). In addition, two pairs of isogenic platinum-resistant cell lines (OV81/CP10 ${ }^{14}$ and A2780/ CP70) were also sensitive to Indomethacin (Figure 3a, right panel). We further confirmed the effects of Indomethacin on cellular survival of the OV81.2 and OV81.2-CP10 cells using clonogenic assays (Figure $3 \mathrm{~b}$ ). Intriguingly, though both the sensitive and resistant cells responded to indomethacin, the platinum-resistant derivatives displayed a more robust induction of $\mathrm{G} 1 / \mathrm{S}$ cell cycle arrest upon treatment with Indomethacin (Figure 3c) and cell death analysis by Annexin-V staining also showed increased cell death in platinum-resistant cells as compared with platinumsensitive cells (Figure $3 d$ ). Interestingly, Indomethacin had minimal effects on non-transformed fallopian tube and ovarian surface epithelium cells at the doses with which it induced robust cell death in ovarian tumor cells (Figure $3 d$ ) suggesting that the pathways targeted by Indomethacin may be critical regulators of EOC pathogenesis and would not affect normal ovarian function. In addition, cleaved-PARP and $\mathrm{Y}-\mathrm{H} 2 \mathrm{AX}$, markers of apoptosis and DNA damage, were greatly elevated in Indomethacin treated OV81.2-CP10 and CP70 cells (Figure 3e).

Indomethacin exerts additive effect with cisplatin in ovarian tumor cells

We next explored the effect of Indomethacin and cisplatin combination on both cisplatin-sensitive and cisplatin-resistant ovarian tumor cells to test the utility of Indomethacin as a potential chemoadjuvant compound. The combination of Indomethacin and cisplatin decreased cell viability more effectively than cisplatin alone in both cisplatin-sensitive and resistant cells (Figure 4a and Supplementary Fig 1a). Isobologram analysis and combination index studies showed that Indomethacin exerts additive effect with cisplatin (combination index 1.0) and decreased IC50 of cisplatin in both cisplatin-sensitive and resistant cells (Figure $4 \mathrm{~b}$ and Supplementary Fig $1 \mathrm{~b}$ ). We further confirmed that cisplatin and Indomethacin combination decreases cell survival using clonogenic assays (Figure 4c). We next investigated the effect of Indomethacin and cisplatin combination on apoptosis in both cisplatin-sensitive and resistant cells. Indomethacin and cisplatin combination induced significantly higher cell death as measured by Annexin-V-PI staining in both cisplatinsensitive (OV81.2) and cisplatin-resistant (OV81.2-CP10 and CP70) 


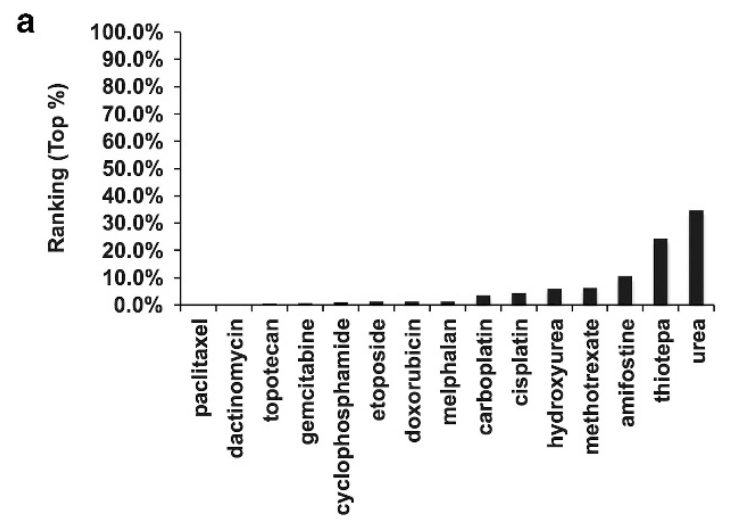

C
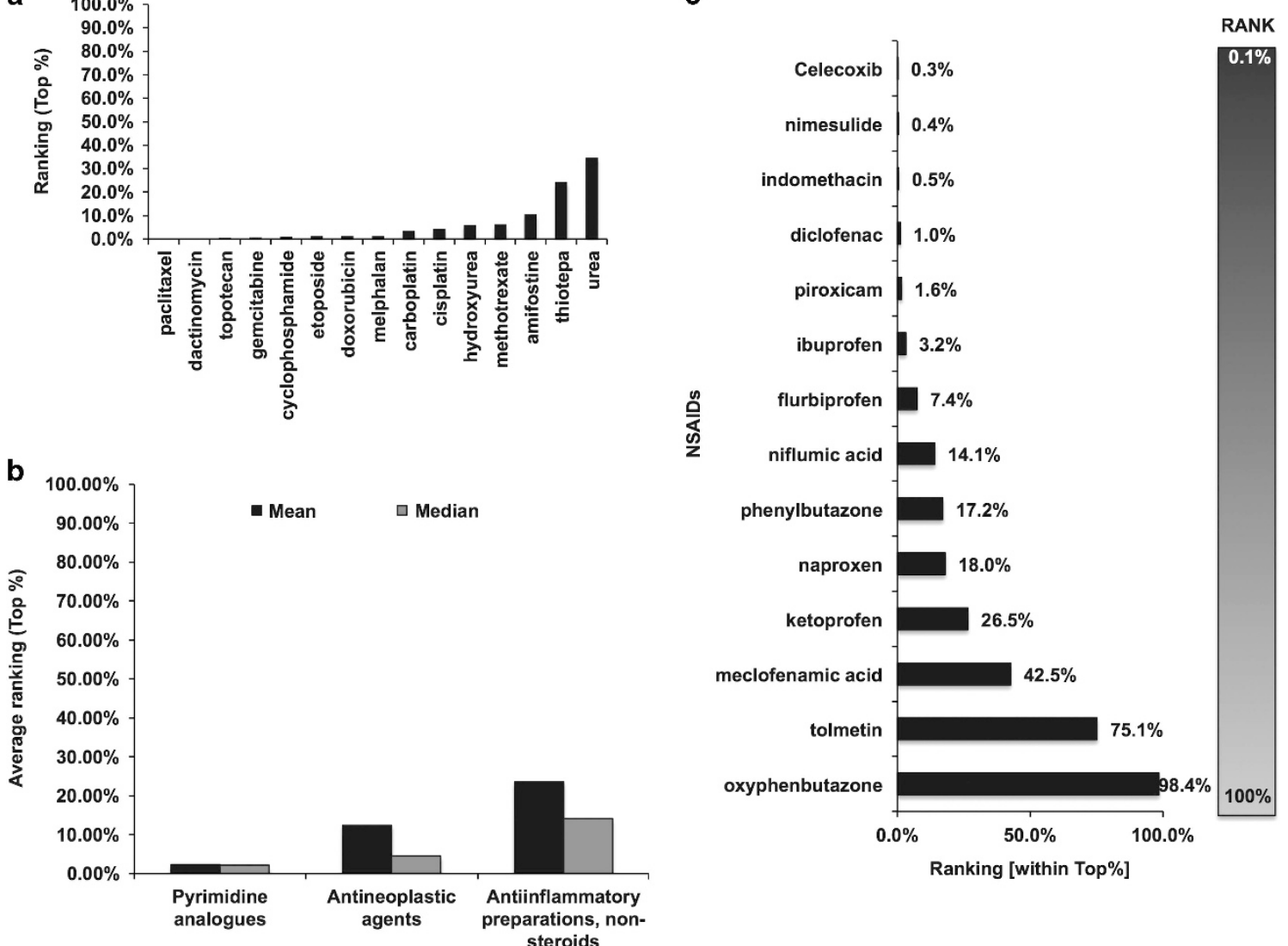

Figure 2. DrugPredict ranked Non-steroidal anti-inflammatory (NSAID) significantly high. (a) Among a total of 6996 prioritized chemicals (including 1096 FDA-approved drugs), the 15 FDA-approved EOC drugs had a median ranking of $1.34 \%$ and mean ranking of $6.44 \%$ (b) Among a total of 882 fourth-level drug classes, 31 classes of drugs ranked significantly higher than random expectation. As expected, anticancer drugs (platinum compounds, pyrimidine analogs and other antineoplastic agents) ranked highest and anti-inflammatories were third highest. (c) List of the highly ranked NSAIDs reveal that celecoxib, nimesulide and indomethacin are the top 3.

cells as compared with the individual drugs alone (Figure 4d). In addition, cleaved-PARP and $\mathrm{\gamma}-\mathrm{H} 2 \mathrm{AX}$ levels were greatly elevated in cisplatin-resistant cells treated with Indomethacin and cisplatin combination as compared with cisplatin alone (Figure 4e). These results unravel a previously unknown possibility that Indomethacin could be potentially employed as a chemoadjuvant to platinum-based treatment regimens in EOC.

Indomethacin downregulates Wnt/ $\mathrm{B}$-catenin signaling in platinum-resistant ovarian tumor cells

NSAIDs that belong to the category of COX-1 inhibitors are reported to function both by COX-1 dependent and COX-1independent mechanisms in functioning as anticancer agents. ${ }^{37}$ COX-1 driven PGE2 is an important constituent of tumors and is implicated in regulation of inflammatory processes driven by tumor microenvironment. ${ }^{38,39}$ However numerous studies have concluded that COX-independent mechanisms could be potentially important in determining the anti-tumor effects of NSAIDs. ${ }^{37,40}$ One such pathway is the $W n t / \beta$-catenin signaling pathway, which is reported to be targeted by NSAIDs in exerting anti-tumor effects especially in colon cancer models. ${ }^{41}$ Also, PGE2, which is the main target of COX-1 inhibitors is a known positive regulator of Wnt signaling. ${ }^{42}$ Furthermore, we recently identified $\beta$-catenin as a critical regulator of platinum resistance through the maintenance of TICs in EOC. ${ }^{36}$ Hence, we hypothesized that downregulation of Wnt/ $\beta$-catenin signaling could be one of the main mechanisms underlying the robust anti-tumor effects of Indomethacin especially in cisplatin-resistant cells. Interestingly, we found that Indomethacin decreased $\beta$-catenin protein level in cisplatin-resistant cells (OV81.2-CP10 and CP70) but not in cisplatin-sensitive cells (OV81.2 and A2780), suggesting that the mechanisms underlying Indomethacin effects could be different in the context of cisplatin-sensitive cells (Figure 5a). Also, Indomethacin did not decrease $\beta$-catenin protein level in nontransformed non-transformed fallopian tube and ovarian surface epithelium epithelial cells (Figure 5a). Furthermore, $\beta$-catenin protein level was greatly reduced upon combination treatment of cisplatin plus Indomethacin as compared with cisplatin alone in cisplatin-resistant cells (Figure $5 \mathrm{a}$ ). In addition, numerous transcriptional targets of $\mathrm{Wnt} / \beta$-catenin signaling implicated in ovarian tumorigenesis were decreased upon Indomethacin treatment (Figure 5b). Specifically, the expression of LEF-1 and LGR5, which are essential targets of Wnt/ $\beta$-catenin pathway and have been recently reported to be highly expressed in the cancer-prone niche in ovarian surface epithelium ${ }^{43}$ as well as in chemoresistant ovarian tumor cells, ${ }^{36}$ were decreased by Indomethacin (Figure $5 \mathrm{~b}$ ). These results suggest that one of the main mechanisms underlying the increased sensitivity of cisplatinresistant cells to Indomethacin and the additive effect of Indomethacin with cisplatin in these cells could be selective inhibition of $W n t / \beta$-catenin signaling in these cells since it is known that $W n t / \beta$-catenin signaling inhibition decreases the survival of cisplatin-resistant cells and induces chemosensitivity in these cells. ${ }^{36}$

As Indomethacin decreased $\beta$-catenin protein expression in cisplatin-resistant cells, we further explored the dependency of Indomethacin effects on $\beta$-catenin, by stably expressing 
a
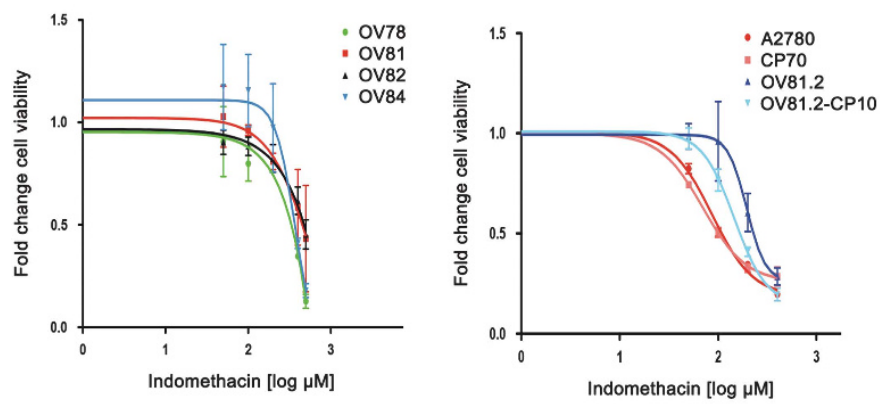

b

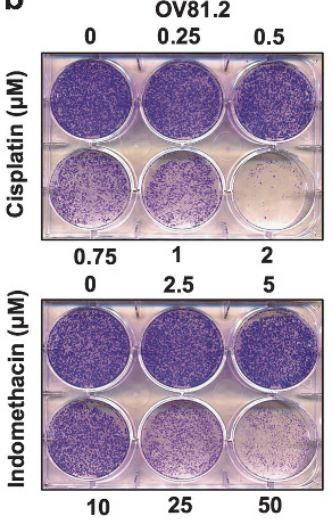

OV81.2-CP10

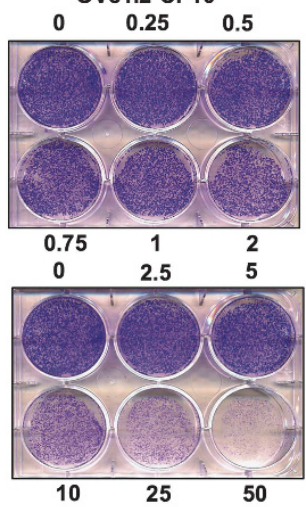

C

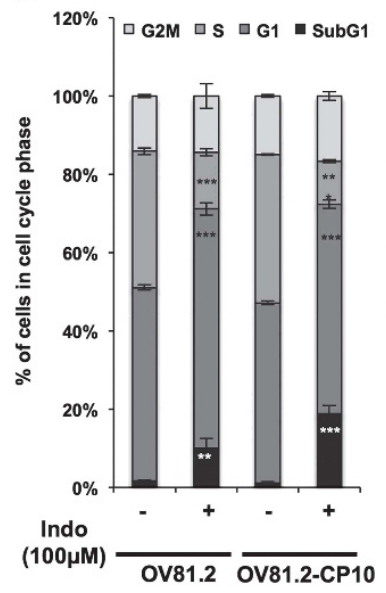

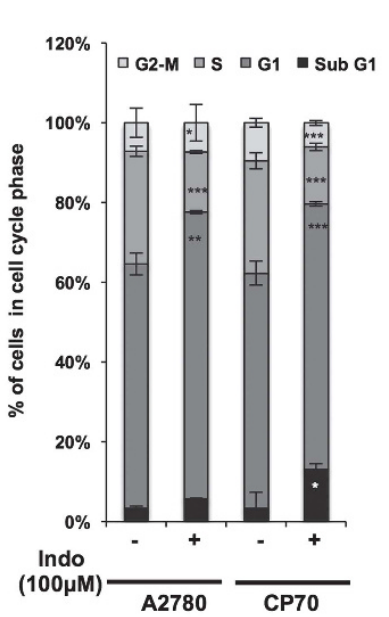

d

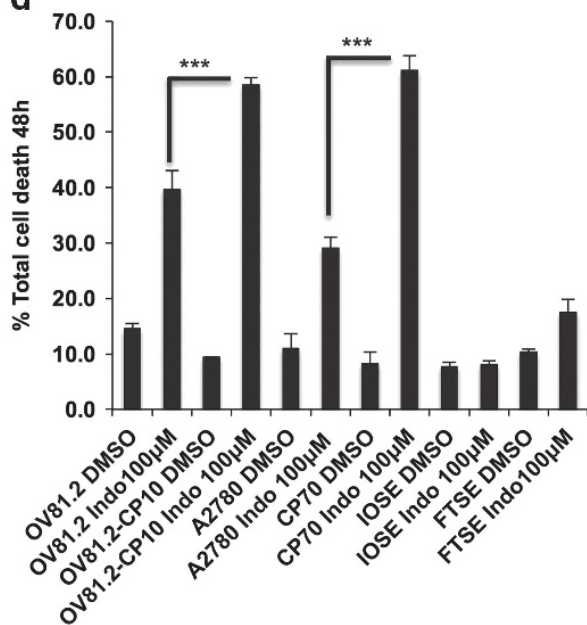

e

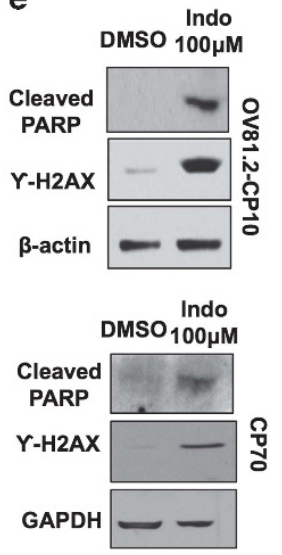

Figure 3. Indomethacin decreases survival of both platinum-sensitive and platinum-resistant ovarian tumor cells. (a) $48 \mathrm{~h}$ MTT assay showing Indomethacin decreases viability in primary epithelial ovarian cancer cells (OV78: Stage IV HGSOC, OV81: Stage IIIC HGSOC, OV82: Stage IIIC low-grade serous EOC, OV84: Stage IIIC HGSOC). (b) Dose-response clonogenics assay on day 7 showing both OV81.2 and OV81.2-CP10 are sensitive to Indomethacin. (c) $48 \mathrm{~h}$ cell cycle analysis showing Indomethacin induces G1 arrest and increased cell death in patient-derived primary platinum-sensitive (OV81.2) and resistant (OV81.2-CP10) cell lines as well as established platinum-sensitive (A2780) and resistant (CP70) cells. (d) $48 \mathrm{~h}$ flow cytometry analysis of Annexin-V PI staining showing robust cell death induced in ovarian tumor cells upon Indomethacin treatment. (e) Western blots showing elevated cleaved-PARP and $\gamma-\mathrm{H} 2 \mathrm{AX}$ protein levels by $24 \mathrm{~h}$ of Indomethacin treatment in CP10 and CP70 ( ${ }^{*} P<0.05,{ }^{* *} P<0.005$ and $\left.{ }^{* * *} P<0.0005\right)$.

non-degradable $\beta$-catenin (which harbors the mutation S33Y) in OV81.2, which we have previously shown induces a cisplatin-resistant phenotype ${ }^{36}$ (Figure $5 c$ ). Indomethacin did not decrease $\beta$-catenin protein expression in $\beta$-catenin-S33Y overexpressed cells (Figure $5 c$ ). Accordingly, overexpressing $\beta$-cateninS33Y protein partially rescued Indomethacin's effects on cell survival and cell death (Figures $5 \mathrm{~d}$ and e). Next, given that $\beta$-catenin downregulation formed the critical component of Indomethacin actions in EOC, we employed loss-of-function assays to assess whether knockdown of $\beta$-catenin further potentiated Indomethacin actions in EOC (Figure 5f). As expected, $\beta$-catenin knockdown potentiated cell death induced by Indomethacin in both OV81.2-CP10 and CP70 (Figure 5g). There was a twofold increase in cell death induced by Indomethacin upon $\beta$-catenin knockdown in both OV81.2-CP10 and CP70 (Figure $5 \mathrm{~g}$ ).

Indomethacin exerts additive effect with cisplatin in ALDH pos ovarian TICS

Aldehyde dehydrogenase 1 (ALDH1) expression is reported to be very high in cancer-prone niche in ovarian surface epithelium, suggesting that it could be involved in early transformation events in EOC. ${ }^{43}$ ALDH pos ovarian tumor cells have been identified as the TICs in ovarian cancer and are reported to be chemoresistant, have high expression of cancer stem cell markers and associated with poor clinical outcome and ALDH pos ovarian tumor cells are being employed as a reliable model to study mechanisms underlying chemoresistance in ovarian TICs. ${ }^{44}$ We have observed that platinum-resistant CP70 have very high ALDH1 expression, ${ }^{36}$ thus we sorted out ALDH-positive cells from CP70 by using ALDEFLOUR flow cytometry assay. These ALDHposCP70 cells exhibited increased expression of cancer stem cell markers such as EpCAM, CD133 and drug resistance markers like ABCG2, exhibited increased tumor sphere formation in limiting dilution tumor sphere formation assays and exhibited increased tolerance to cisplatin (Figures $6 a$ and b). ALDH1 has also been recently identified as a direct target of $\beta$-catenin in ovarian tumor cells, ${ }^{45,36}$ suggesting that $\mathrm{Wnt} / \beta$-catenin signaling could have an important role in regulating TIC functions in EOC. Hence, we next investigated whether Indomethacin can affect ALDHposCP70 TICs. We found that Indomethacin induced G1/S cell cycle arrest, and decreased tumor sphere formation (Figures $6 c$ and $d$ ). Furthermore, Indomethacin decreased the expression of $\mathrm{Wnt} / \mathrm{\beta}$ - catenin transcriptional targets that regulate stem-like properties TCF7, LGR5, CD24 and EpCAM (Figure 6e).

We next examined the combinatorial effects of cisplatin and indomethacin in the ALDH pos ovarian TICs, similar to the 
a

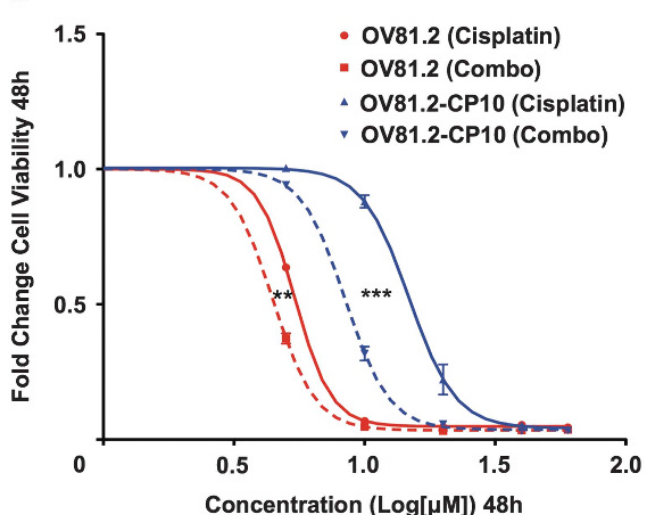

b

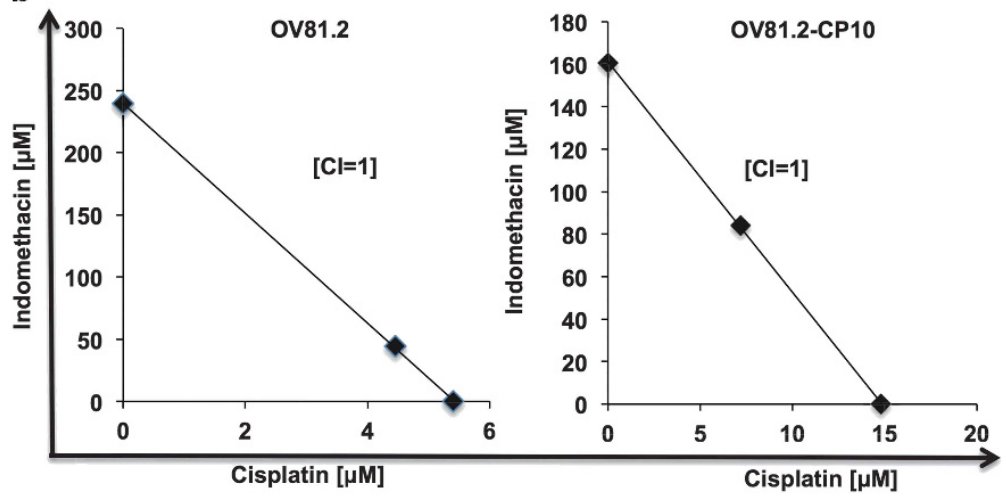

C
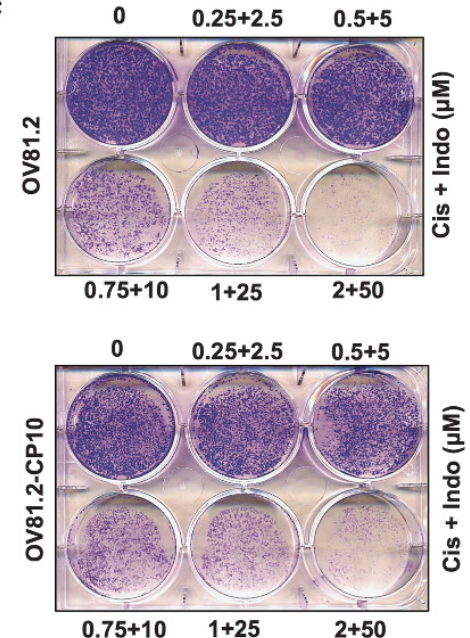

d

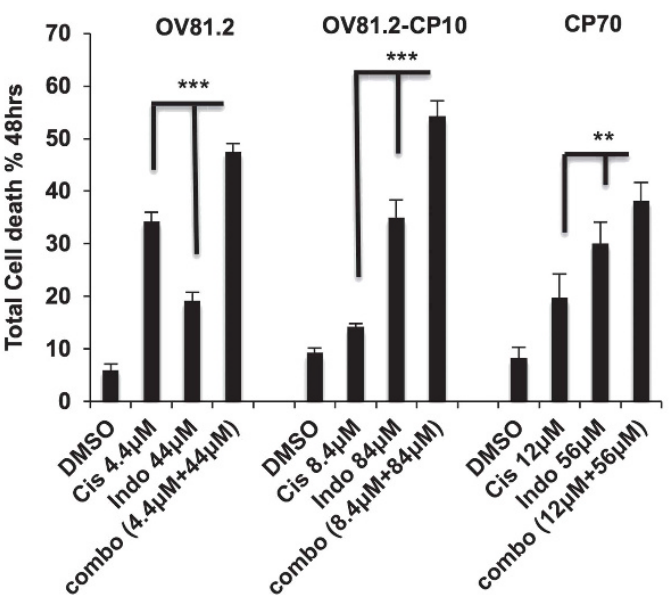

e
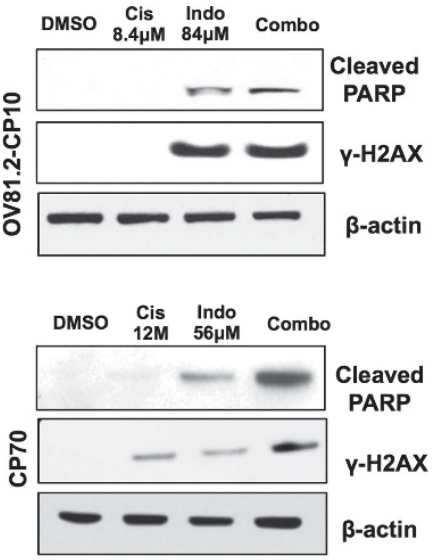

Figure 4. Indomethacin exerts additive effect with cisplatin in ovarian tumor cells. (a) $48 \mathrm{~h}$ MTT analysis of OV81.2 and OV81.2-CP10 cells treated with increasing doses of cisplatin and cisplatin plus Indomethacin. (b) Isobologram analysis of the MTT data of Indomethacin cisplatin combination treatment in OV81.2 and OV81.2-CP10 cells showing additive effect of the combination of the two drugs. (c) Dose-response clonogenics assay on day 7 showing effect of Indomethacin and cisplatin combination on cell survival in OV81.2 and OV81.2-CP10. (d) $48 \mathrm{~h}$ Flow cytometry analysis of Annexin- V PI staining showing increased cell death upon Indomethacin and cisplatin combination treatment with the corresponding isobologram doses in OV81.2, OV81.2-CP10 and CP70. (e) Western blots showing elevated cleaved-PARP and $\gamma$-H2AX protein levels upon $24 \mathrm{~h}$ Indomethacin and cisplatin combination treatment with the corresponding isobologram doses in OV81.2-CP10 and CP70 ${ }^{* *} P<0.005$ and $\left.{ }^{* * *} P<0.0005\right)$.

differentiated cells isobologram analysis showed that Indomethacin exerts additive effect with cisplatin in ALDH pos ovarian TICs and decreased the IC50 of cisplatin by $\sim 50 \%$ in these cells (Figure 6f). Consistent with these findings, the combination of indomethacin and cisplatin induced robust cell death and decreased cell survival in both ALDH pos and ALDH neg CP70 cells (Figure $6 \mathrm{~g}$ ). In addition, cleaved-PARP and $\mathrm{\gamma}$-H2AX levels were greatly elevated in Indomethacin and cisplatin combination treated cells as compared with individual drugs alone (Figure 6h) and as observed with CP10 and CP70, $\beta$-catenin protein level was greatly reduced upon combo treatment in these TICs as compared with cisplatin alone (Figure $6 \mathrm{~h}$ ). These results suggest that Indomethacin could be used as a chemoadjuvant in EOC to eradicate both non-TICs (ALDH neg) and TICs (ALDH pos) in ovarian cancer, thus potentially overcoming the barrier of tumor recurrence in ovarian cancer.

\section{DISCUSSION}

There is an urgent need for new treatment options for patients with recurrent, platinum-resistant EOC. TICS are thought to be the root cause of tumor recurrence and chemoresistance in EOC, and platinum-based treatment regimens are known to be ineffective against these TICs. ${ }^{36}$ Existing therapies to treat EOC do not eradicate the TICS thus aiding in tumor relapse and drug resistance. A greater understanding of the molecular alterations driving the progression and treatment resistance of the most common and lethal form of EOC, HGSOC will allow for the development of rationally designed targeted molecular therapies to treat the underlying drivers of the disease. At present, there are no standard therapeutic options for patients with recurrent chemotherapy resistant tumors.

DrugPredict is a general approach for drug discovery and repurposing by innovatively interrogating human genes to corresponding mouse functional phenotypes. There are several major differences between DrugPredict in this study and our previously published methods: ${ }^{7,17}$ (a) DrugPredict is more efficient and we applied it to search drug candidates (both repositioned drugs and chemicals) for hundreds of thousands of chemicals rather then the previously published method which was less efficient and only applied to <2000 FDA-approved drugs, (b) DrugPredict uses more stricter criteria in calculating profile similarities in order to achieve high specificity and our previously published approach which used semantic distance tends to 

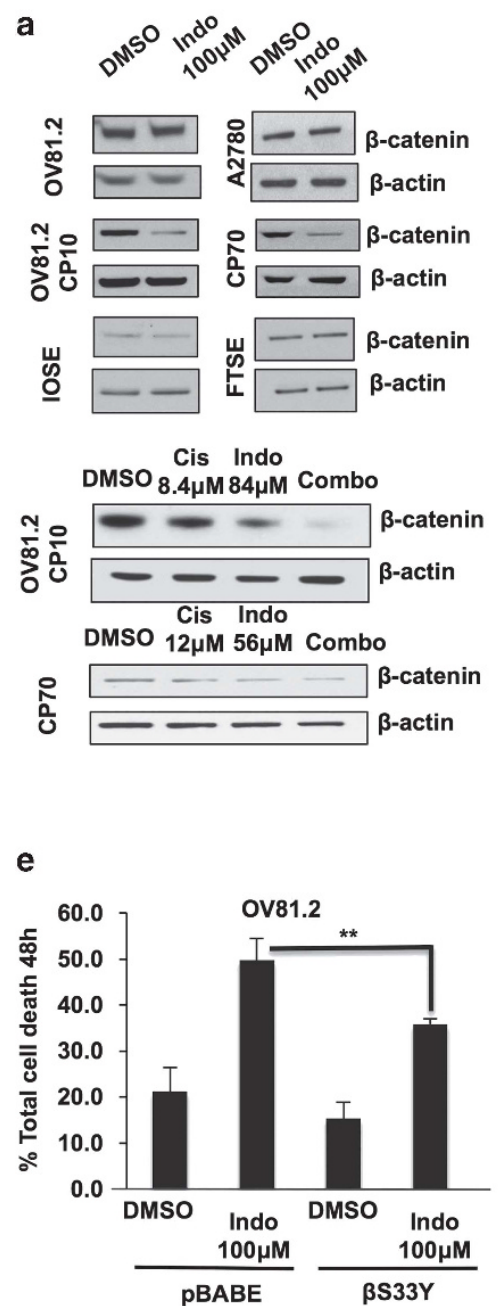

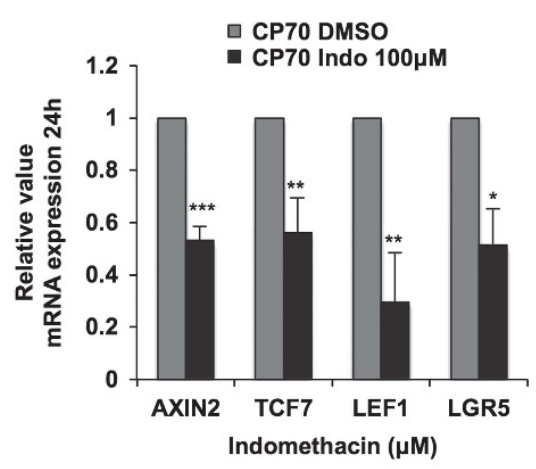

C
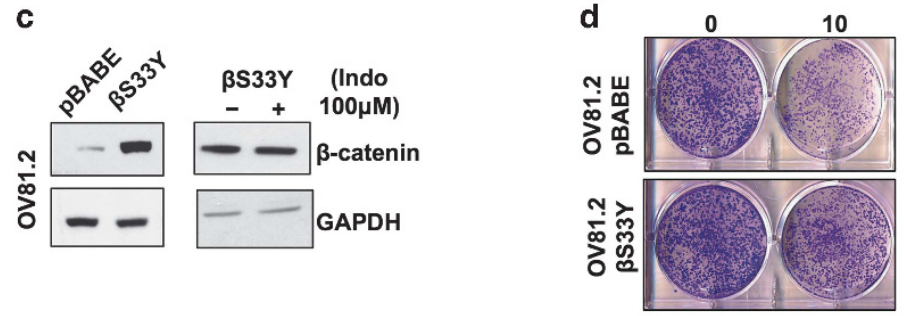

f

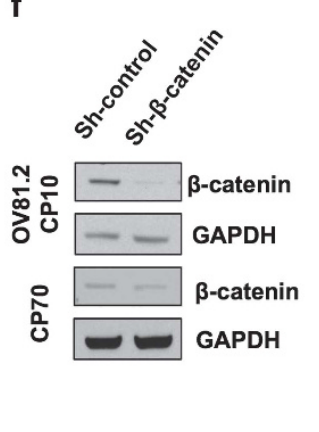

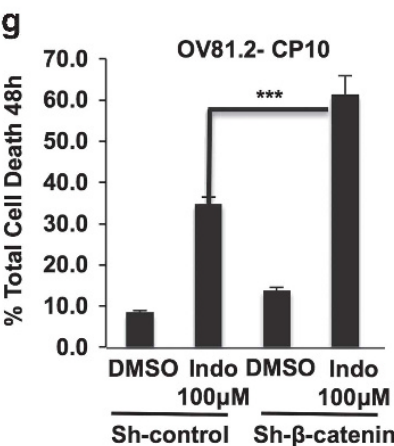

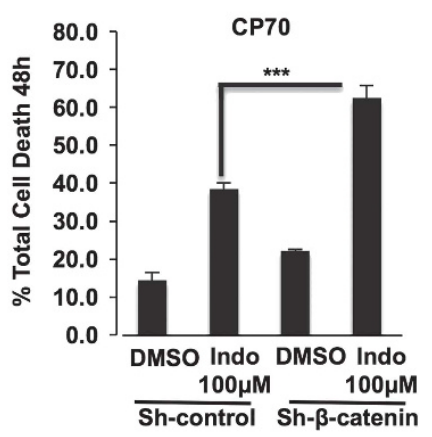

Figure 5. Indomethacin downregulates Wnt/ $\beta$-catenin signaling in platinum-resistant ovarian tumor cells. (a) Western blot showing robust decrease in $\beta$-catenin protein level by $24 \mathrm{~h}$ upon treatment with Indomethacin in OV81.2-CP10 and CP70 cells and enhanced $\beta$-catenin downregulation in cisplatin plus Indomethacin combination treated OV81.2-CP10 and CP70 cells as compared with individual drug treatment alone $24 \mathrm{~h}$. (b) Real-time PCR analysis $24 \mathrm{~h}$ showing Indomethacin decreases mRNA expression of $\beta$-catenin transcriptional targets AXIN2, TCF7, LEF-1 and LGR5 in OV81.2-CP10 and CP70. (c) Western blot showing overexpression of non-degradable $\beta$-catenin ( $\beta$-S33Y) in OV81.2 (left) and effect of Indomethacin on $\beta$-catenin protein expression in OV81.2- $\beta$ - S33Y cells (right). (d) clonogenics assay on day 7 showing $\beta$-S33Y overexpression partially rescues the effect of Indomethacin on cell survival in OV81.2. (e) $48 \mathrm{~h}$ Annexin-V PI staining flow cytometry analysis showing that $\beta$-S33Y overexpressing OV81.2 cells are more tolerant to Indomethacin induced cell death. (f) Western blot confirming lentiviral shRNA mediated $\beta$-catenin knockdown. (g) $48 \mathrm{~h}$ analysis of Annexin-V PI staining showing increased cell death upon Indomethacin treatment in OV81.2-CP10 and CP70 sh- $\beta$-catenin cells compared with sh-scram control $\left({ }^{*} P<0.05,{ }^{* *} P<0.005\right.$ and. $\left.{ }^{* *} P<0.0005\right)$.

introduce more false positives and (c) last, the previously published method directly used the list of phenotypes associated with disease genes to construct phenotype profiles, whereas in this current study in order to increase the specificity of phenotype profile and repositioned drugs for ovarian cancer, then we first performed analysis to identify phenotypes that are significantly enriched for ovarian cancer and then used these significantly enriched phenotypes to construct phenotype profile. Through this new method we have now demonstrated DrugPredict's utility in drug repurposing for EOC and uncovered that FDA-approved NSAID Indomethacin represents a potent new compound to treat HGSOC. Our results show that Indomethacin inhibits cell proliferation, cell survival and induces robust cell death in cisplatin-resistant cells and ovarian TICs through the downregulation of $\beta$-catenin. Furthermore, expression of transcriptional targets of $\beta$-catenin that are directly implicated in ovarian tumorigenesis and platinum resistance were greatly decreased by Indomethacin. In addition, we found that Indomethacin exerted additive effects with cisplatin in both cisplatin-sensitive and cisplatin-resistant ovarian tumor cells. Overall, our results identify a hitherto unknown application of Indomethacin as a potential chemoadjuvant in EOC and also provides with a good platform to extend this observation to other NSAIDs.

Although the Wnt/ $\beta$-catenin signaling pathway has been extensively studied in many cancers, we have only recently shown that this pathway drives platinum resistance and the maintenance of TICS in ovarian cancer. ${ }^{36}$ We have shown that Wnt/ $\beta$-catenin signaling confers survival advantage in response to long-term cisplatin treatment and platinum resistance in ALDH pos ovarian TICs is driven by $\beta$-catenin. ${ }^{36}$ Hence, drugs affecting TICs through downregulation of Wnt/ $\beta$-catenin signaling could form excellent chemoadjuvant combination with cisplatin where in both differentiated tumor cells and $\beta$ - catenin driven TICs can be efficiently eradicated thus preventing tumor recurrence and improving survival outcome in EOC. Interestingly, Indomethacin did not decrease $\beta$ - catenin protein expression in cisplatinsensitive cells that were sensitive to the drug and exhibited additive cell death upon combination with cisplatin. Cisplatin- 
a

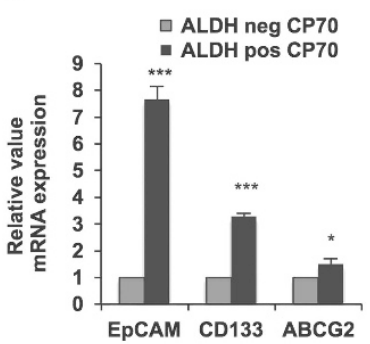

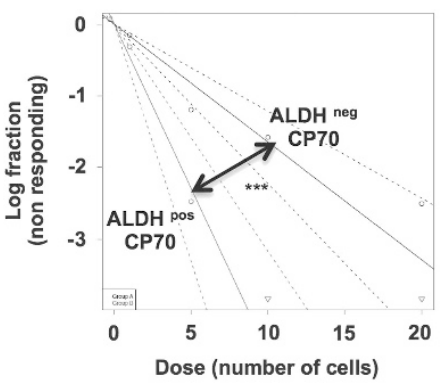

b

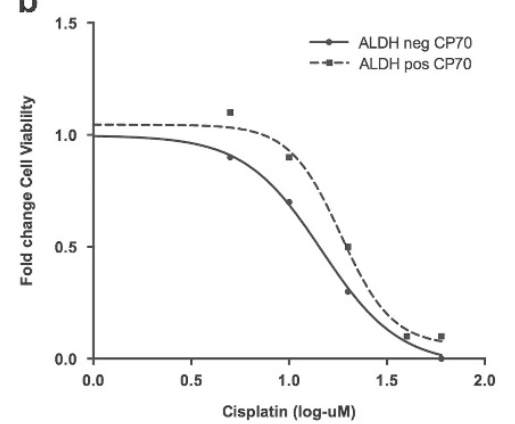

C

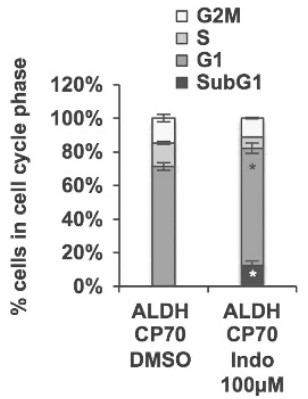

d

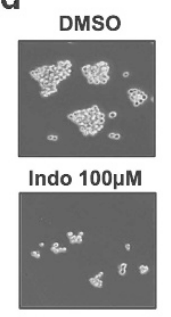

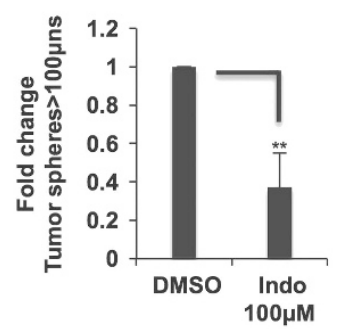

g

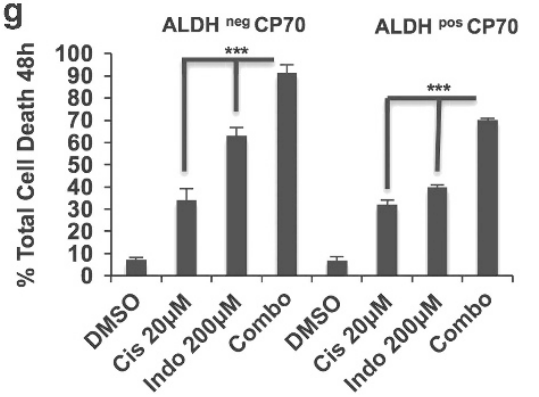

e
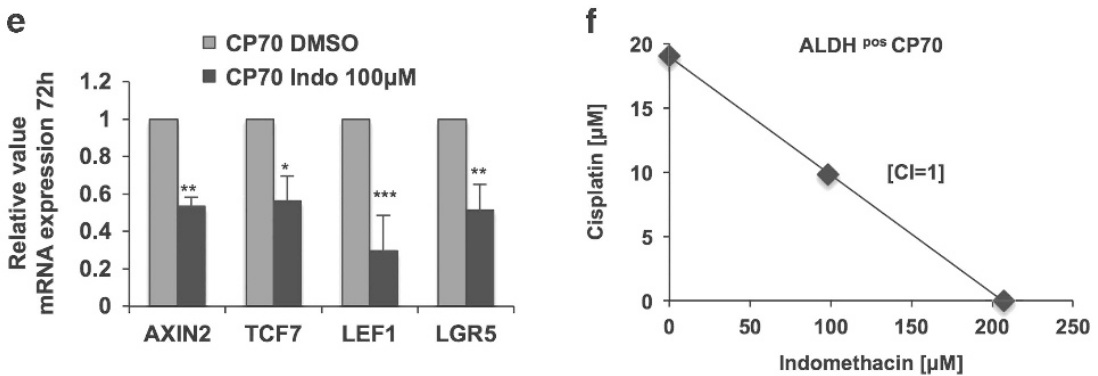

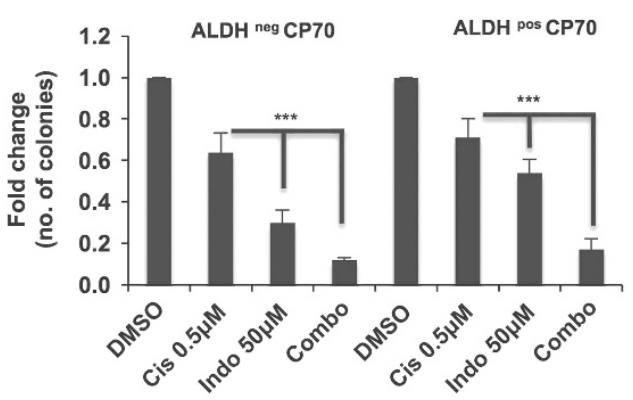

h
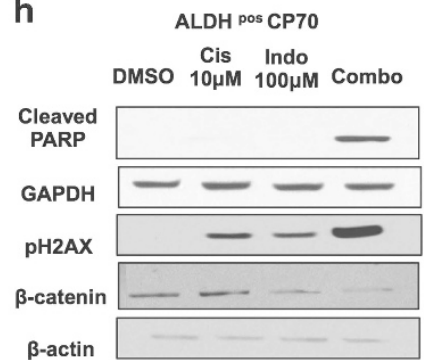

$\beta$-actin

Figure 6. Indomethacin exerts additive effect with cisplatin in ALDH pos ovarian tumor-initiating cells (TICs). (a) Real-time PCR analysis showing increased mRNA expression of cancer stem cell markers EpCAM, CD133 and drug resistance marker ABCG2 in ALDH-positive CP70 cells (left) limiting dilution tumor sphere formation analysis showing increased tumor sphere formation ability in ALDH pos CP70 cells (right). (b) $48 \mathrm{~h}$ MTT analysis showing increased tolerance to cisplatin in ALDH pos CP70 cells. (c) PI staining cell cycle flow cytometry showing cell cycle arrest in G1 phase and increased SubG1 phase induced by Indomethacin in ALDH pos CP70 cells. (d) $\times 10$ light microscopy images and $10 \times 10$ stitch imaging with integrated metamorph software analysis showing decreased tumor sphere formation by day 6 upon Indomethacin treatment. (e) Real-time PCR analysis $72 \mathrm{~h}$ showing Indomethacin decreases the mRNA expression of $\beta$-catenin driven cancer stem cell transcriptional targets in ALDH pos CP70 cells. (f) Isobologram analysis showing additive effect of Indomethacin cisplatin combination in ALDH pos CP70 cells. (g) $48 \mathrm{~h}$ Annexin-V PI staining flow cytomery analysis showing increased cell death upon Indomethacin plus cisplatin combo treatment as compared with individual drugs alone in both ALDH neg CP70 (left) and ALDH pos CP70 cells (right) clonogenics assay on day 7 showing greater decrease in cell survival upon Indomethacin plus cisplatin combo treatment as compared with individual drugs alone in both ALDH neg CP70 and ALDH pos CP70 cells (right). (h) Western blots showing increased cleaved-PARP and $\gamma$-H2AX protein levels and decreased $\beta$-catenin protein level in Indomethacin plus cisplatin combo treated cells as compared with individual drugs alone treated ALDH pos CP70 cells $\left({ }^{*} P<0.05,{ }^{* *} P<0.005\right.$ and $\left.{ }^{* * *} P<0.0005\right)$.

sensitive cells are known to exhibit less stem-like properties by virtue of which they are chemosensitive and also exhibit low activity of Wnt/ $\beta$-catenin signaling. ${ }^{36}$ Hence, the mechanisms underlying effects of Indomethacin in cisplatin-sensitive cells could be different from cisplatin-resistant cells and could potentially involve non-TIC related mechanisms. As Indomethacin induced G1/S cell cycle arrest and DNA damage in ovarian tumor cells and cisplatin also induces DNA damage and S phase arrest, cell cycle related mechanisms could underlie the effects of Indomethacin in these cells. Further understanding of mechanisms underlying Indomethacin actions in cisplatin-sensitive cells would be important in realizing the application of NSAIDs as chemoadjuvants in the initial regimens of platinum therapy in patients newly diagnosed with ovarian cancer. Indomethacin has been reported to be efficient in decreasing tumor burden in vivo in ovarian cancer but this study looked at the effect of
Indomethacin on a tumor cell line that does not truly reflect HGSOC pathogenesis in a clinical scenario. ${ }^{46}$ Also, the utility of Indomethacin as a potential chemoadjuvant was not tested in this study. Hence, it is important to test the efficacy of Indomethacin in patient-derived xenograft models of HGSOC to further validate the possibility of employing it as a chemoadjuvant tool in EOC. Our data form a good basis and provides with rationale to test NSAIDs in such platinum-resistant patient-derived xenograft models and potentially expanding this to patient clinical trials.

In this study we demonstrate DrugPredict's utility in drug repurposing for EOC however we did not tailor the system for EOC, therefore, we expect that DrugPredict would be equally effective in identifying drug candidates for other diseases. Nevertheless, there are some limitations including the inability to differentiate the associations ('Treat or 'Cause') between the input disease and drug-associated phenotypes. For example, 
among top-ranked phenotypes for indomethacin, the phenotype 'decreased inflammatory response' is related to drug treatment since we know that indomethacin is an anti-inflammatory drug. Two other phenotypes 'increased myocardial infarction size' and 'increased hepatocyte apoptosis' are also significantly enriched. Based on our knowledge that indomethacin can cause cardiovascular thrombotic events and hepatotoxicity, we know that the associations between indomethacin and these two phenotypes are related to drug toxicities. However, we currently lack sufficiently fine-grained data to automatically classify the associations between drugs and phenotypes into 'Treatment' or 'Toxicity'. A drug (for example, indomethacin) often up-/downregulates many genes at the same time. The mutations of each gene in mouse models can cause many phenotypes. Currently, it is challenging to develop algorithms to automatically summarize the collective effects of drugs' genes on a specific phenotype and classify them into 'Treatment' or 'Toxicity'. Semantically classifying the associations between drugs and phenotypes is important for both drug discovery and drug toxicity prediction, which will be one of the focuses of our future work. Second, although mice and humans share virtually the same set of genes, the expression and function of the genes may be different in mouse models and humans. For example, a recent study by the Mouse ENCODE Consortium showed that there are considerable differences between human gene and mouse gene expression profiles however, the phenotype-level divergence between human genes and mouse genes may be less prominent. ${ }^{47}$ Though our study in prioritizing and validating indomethacin for EOC treatment provides further support this strategy, due to the inherent difference between human and mouse models, the functions of a gene in human may not completely conserved in mouse models. To summarize, we have developed a novel drug discovery strategy driven by the novel computational drug-repurposing platform DrugPredict coupled with validation in ovarian cancer patientderived xenograft models and have identified Indomethacin as a novel potential chemoadjuvant in EOC. Our study highlights the importance of applying computational drug-repositioning approaches to TCGA datasets in cancer to reliably identify novel drug candidates that could be efficiently translated to clinical settings in a cost-effective manner. Furthermore, given the relationship between the two drugs use of the combination treatment may also reduce the typical toxic side-effects seen with platinum-based therapies because a lower concentration of cisplatin can be used.

\section{MATERIALS AND METHODS}

Performing DrugPredict analysis

Obtaining HGSOC genes from TCGA. The microarray data for whole-genome mRNA expression were downloaded from TCGA data portal (https://tcga-data.nci.nih.gov/). This data set contains 587 clinically annotated grade I-IV ovarian cancer samples (6 G1, $78 \mathrm{G} 2,487 \mathrm{G} 3$ and $1 \mathrm{G} 4$ ) and 8 normal fallopian tube samples. A total of 17814 mRNA expression profiles were measured in the Agilent $244 \mathrm{~K}$ platform. Data were lowess normalized and log2 transformed within array. Quantile approach was used for between-array normalization. A total of 1531 deferentially expressed genes between normal (8 samples) and grade III (487 samples) were identified using limma (R package) with adjusted $P$-value $<0.001$. The clinical characteristics was also described in a recently published study. ${ }^{48}$

Construction HGSOC-specific mouse mutation phenotype profile. We downloaded the genotypes and Mammalian Phenotype Annotations data from $\mathrm{MGD}^{26}$ and mapped the mouse genes to their human homologs using human-mouse homolog mapping data from the MGD (for example, TP53->Trp53). We obtained a total of 178626 human gene-mouse mutational phenotype pairs from MGD. One such pair is 'TP53 (tumor protein p53)-increased ovarian carcinoma incidence'. We then directly linked the 1531 HGSOC genes to mouse mutational phenotypes using the 178626 human gene-mouse mutational phenotype mappings. A total of 4627 mouse mutational phenotypes are mapped to HGSOC genes. For each of these mapped phenotypes, we assessed the probability of this phenotype being associated with HGSOC genes as compared with the same number of randomly selected genes. We repeated the random process 1000 times and perform a $t$-test to assess the enrichment significance between each phenotype and HGSOC genes (the list of significantly enriched phenotypes for HGSOC genes is provided as supplementary materials).

Construction of mutational phenotype profiles for FDA-approved drugs. We obtained chemical-associated genes from CTD (Comparative Toxicogenomics Database).$^{49}$ CTD provides information about interactions between chemicals and gene products, as well as their relationships to diseases. Core CTD content includes chemical-gene, chemical-disease and gene-disease interactions that were manually curated from the literature. We used the chemical-associations as the resources of drug genetics. We obtained a total of 973296 chemical-gene interactions from CTD, including 386190 interactions in humans. These 386190 chemical-gene associations include 7570 chemicals/drugs and 20116 genes. An example of such chemical/drug-gene association is: 'indomethacin-ABCC1', where indomethacin results in decreased expression of ABCC1 mRNA. For each of the 7570 chemicals/drugs (for example, indomethacin), DrugPredict first obtained its associated genes from CTD. It then linked these genes to their mouse mutational phenotypes through the 178626 human genemouse mutational phenotype mappings that we obtained from MGD. The mouse mutational phenotype profile for each chemical was constructed in the same way as the HGSOC-specific phenotype profile (describe above). For example, we obtained a total 329 indomethacin-associated genes from CTD. We identified 1961 mouse mutational phenotypes that are significantly enriched for these 329 genes. For example, the phenotype 'decreased tumor incidence' is associated with 18 indomethacin-associated genes, and it represents a sevenfold enrichment as compared with random expectation. The list of phenotypes that are significantly enriched for indomethacin is provided as supplementary materials.

As our goal is to find repositioning candidates from FDAapproved drugs, we filtered chemicals from CTD using 1648 FDAapproved drugs derived from DrugBank. ${ }^{50}$ We used CTD instead of DrugBank to obtain drug-gene associations as CTD contains both on-target and off-target gene associations for FDA-approved drugs. As the main goal of our drug-repurposing strategy is to find surprising/unexprected off-target effects of FDA-approved drugs in diseases, we used CTD instead of DrugBank, the latter contains mainly drugs' on-target information. For example, Drugbank contains eight genes for indomethacin (for example, PTGS1, PPARG, GRP44). CTD contains 621 indomethcin-gene associations.

Prioritization drugs based on the similarities between HGSOC-specific phenotype profile and drug-associated phenotype profiles. Using the HGSOC-associated phenotype profile (1426 phenotypes) and drug-associated phenotype profiles as inputs (for example, 1961 phenotypes for indomethacin) as inputs, DrugPredict calculated the similarity between HGSOC and drugs using the Jaccard coefficient. Jaccard similarity coefficient is a statistic used for measuring the similarity between finite sample sets, and is defined as the size of the intersection divided by the size of the union. For example, a total of 620 phenotypes are common for HGSOCspecific phenotype profile and indomethacin phenotype profile. The Jaccard coefficient is $0.224(620 /(1426+1961-620))$ between HGSOC and indomethacin. On the other hand, a total of 294 
412

phenotypes are common between HGSOC and ibuprofen profiles (1039 phenotypes). The Jaccard coefficient is 0.136 (294/(1426 +1039-296)) between HGSOC and ibuprofen. Based on the Jaccard coefficient values, DrugPredict ranked indomethacin higher than ibuprofen. PhenoPredict built phenotype profiles for all drugs and then calculated the Jaccard coefficients for each drug. The output of DrugPredict is a list of drugs ranked based on the Jaccard coefficients.

Evaluation of DrugPredict. We evaluated the performance of DrugPredict in OC drug discovery using 16 FDA-approved OC drugs (http://www.drugs.com/condition/ovarian-cancer.html). We calculated the recall, which measured how many of these 16 drugs DrugPredict could find and the mean and median rankings of these FDA-approved drugs among all prioritized drugs. The mean ranking of $1 \%$ means that these drugs ranked at top $10 \%$ on average. We also calculated the $P$-value of the mean ranking as compared with random expectation.

Analyze repositioned drug candidates. We then evaluated which categories of drugs ranked significantly highly. We classified drugs based on the ATC classification system. ATC system consists of 13 first-level codes or classes, 94 second-level codes, 267 third-level codes, 882 fourth-level codes and 4580 fifth-level codes, which are individual drugs. In our study, we used both third and fourth-level ATC codes for drug classification. For each category, we calculated the mean ranking and median ranking of drugs in the category. The significance was calculated using $t$-test to assess whether the drug class ranked significantly higher than random. For example, there are a total of seven pyrimidine analogs among our prioritized drugs with a mean ranking of $2.26 \%$ and median ranking of $2.09 \%$, which is significantly higher than random expectation ( $P$-value: $1.74 \mathrm{E}-8)$.

\section{Cell culture and reagents}

A2780 (cisplatin-sensitive) and CP70 (isogenic cisplatin-resistant to A2780) cells were obtained from Dr Paul Modrich (Duke University). All cells used were cultivated in DMEM media supplemented with $10 \%$ FBS and $1 \%$ Penicillin-streptomycin (recommended media). Cells were cultured in $10 \mathrm{~mm}$ plates in a humidified atmosphere $\left(5 \% \mathrm{CO}_{2}\right)$ at $37^{\circ} \mathrm{C}$. At $70-90 \%$ confluence, trypsin $(0.25 \%) /$ ethylenediaminetetraacetic acid solution was used to detach the cells from the culture plate for passaging and used for further experiments until passage 20. Primary patient cell lines were generated from tumors or ascites isolated from patients diagnosed with histologically confirmed HGSOC. Informed consents were obtained from all patients. All experiments were performed in accordance with relevant guidelines and policies of University Hospitals Case Medical Center (Cleveland, $\mathrm{OH}, \mathrm{USA}$ ) and approved by the University Hospitals Case Medical Center IRB committee. The platinum-resistant derivative of OV81.2 namely, CP10 was generated by propagating OV81.2 in the presence of cisplatin for 10 passages. $^{36}$ MTT, Clonogenics and Annexin-V PI assays confirmed the resistance of these cells to cisplatin. ${ }^{36}$ Cisplatin was purchased from Mount Sinai Hospital Pharmacy. Indomethacin was purchased from Sigma-Aldrich (St Louis, MO, USA).

\section{Generation of stable cell lines}

Several stable cell lines were generated for this study and the same protocol was followed as described below. $\beta$-catenin lentiviral shRNA plasmid (Addgene plasmid 18803 deposited by Dr Bob Weinberg's laboratory) and the corresponding pLKO.1 control plasmid (Addgene plasmid 8453 deposited by Dr Bob Weinberg's laboratory) were acquired from Addgene. For lentiviral transfection with the constructs, Lenti Starter Kit (System Biosciences, Palo Alto, CA, USA) was used. In brief, 3M 293T cells were plated in $10-\mathrm{cm}$ plate with antibiotic-free media. At $50-70 \%$ confluence, $2 \mu \mathrm{g}$ of plasmid and $10 \mu \mathrm{g}$ of pPACKH1-plasmid mix were co-transfected with Lipofectamine 2000 (Invitrogen, Carlsbad, CA, USA) following manufacturer's protocol. Forty-eight hours later, virus particles were harvested and precipitated. Target cells were transduced by plating 100000 cells/well in a six-well plate with virus particles and $4 \mu \mathrm{g} / \mathrm{ml}$ polybrene and analyzed $72 \mathrm{~h}$ later. $\beta$-catenin-S33Y retroviral plasmid was a kind gift from Dr Ken-Ichi Takemaru (Stony Brook university). For retroviral transfections, retrovirus was generated by co-transfection of the constructs with packaging plasmids into Phx cells. Target cells were transduced as described above.

RNA extraction and Real-time PCR

Total RNA was extracted using the Total RNA Purification plus Kit (Norgen Biotek, Thorold, ON, Canada) according to manufacturer's instructions. For mRNA analysis, cDNA sysnthesis from $1 \mu \mathrm{g}$ of total RNA was done using the Transcriptor Universal cDNA Master kit (Roche, Indianapolis, IN, USA). SYBR green-based Real-time PCR was subsequently performed in triplicate using SYBR green master mix (Roche) on the Light Cycler 480 II real-time PCR machine (Roche).

\section{Immunoblotting}

Whole-cell protein extracts were probed with antibodies against $\beta$-catenin (1:500) (Cell Signaling, Danvers, MA, USA), cleaved-PARP (1:250) (Promega, Fitchburg, WI, USA), phospho-H2AX (1:250) (Cell signaling) and GAPDH (1:1000) (Santa Cruz, Dallas, TX, USA). Membranes were exposed using LumiLight or LumiLightplus (Roche) method following manufacturer's instructions.

\section{Cell Viability assays}

Cells were plated in 12-well plate at 50000 cells/well and treated the next day with the corresponding drugs. After indicated time points, cells were then incubated with 3-(4,5-Dimethylthiazolyl) for $2 \mathrm{~h}$ and absorbance was measured at $600 \mathrm{~nm}$. Isobologram analysis was done using graph pad prism software.

\section{Clonogenics assay}

Cell survival was assessed through seeding 2000 cells/well in a sixwell plate and treated with indicated doses drugs every 3 days for 7 days. On day 7 , cells were fixed in a $10 \%$ acetic acid/10\% methanol (in $\mathrm{diH}_{2} \mathrm{O}$ ) solution and stained with $1 \%$ crystal violet (in methanol) after 7 days of growth. Colonies were counted using Image J.

\section{Annexin-V/PI staining}

Cells were plated 100000 cells/well in a six-well plate. The next day, cells were treated with drug and harvested after indicated time points. Annexin-V/PI Staining was done using the FITC Annexin-V Apoptosis Detection Kit II (BD Pharmigen, Billerica, MA, USA). FACS data were acquired using the Coulter Epics XL machine.

\section{PI staining}

Cells were plated 100000 cells/well in a six-well plate. The next day, cells were treated with drug and harvested after indicated time points and stored in $70 \%$ ethanol at $-20{ }^{\circ} \mathrm{C}$ until staining. For FACS, cell lysates were stained with $50 \mu \mathrm{g} / \mathrm{ml}$ propidium iodide and $1 \mathrm{mg} / \mathrm{ml}$ RNAse, incubated in dark for $30 \mathrm{~min}$ and were resuspended in $200 \mu \mathrm{l}$ phosphate-buffered saline. Data were acquired with Coulter Epics XL machine and analyzed using FlowJo 10.0.6 software.

\section{Tumor sphere formation}

For tumor sphere formation assays, 1000 cells per $\mathrm{ml}$ were plated in $2 \mathrm{ml}$ in six-well ultra-low attachment plate (Corning, Corning, NY, USA) in MammoCult medium (Stem cell Technologies, 
Cambridge, MA, USA) and after the indicated timepoint, $10 \times 10$ stitch imaging was done at $\times 10$ (100 random images acquired) using an Retiga Aqua Blue camera (Q Imaging, Vancouver, BC USA) connected to a Leica DMI6000 inverted microscope. Individual images were taken and then a composite image was generated using the scan slide function in Metamorph Imaging Software (Molecular Devices, Downington, PA, USA). Subsequent integrated analysis also used Metamorph software. Limiting dilution tumor sphere formation assay was done as previously described. ${ }^{36}$

\section{Flow cytometry analysis and sorting}

For ALDH-based sorting, ALDH assay was done using the ALDEFLUOR kit as per the protocol instructions (Stem cell Technologies) and ALDH-positive and -negative cells were FACS sorted using BD FACS Aria II. For ALDH activity assessment the same kit was used and the data were acquired with Coulter Epics $\mathrm{XL}$ machine.

\section{Statistical analysis}

Unless otherwise noted, data are presented as mean \pm s.d. from three-independent experiments, and Student's $t$-test (two-tailed) was used to compare two groups $(P<0.05$ was considered significant) for independent samples.

\section{CONFLICT OF INTEREST}

The authors declare no conflict of interest.

\section{ACKNOWLEDGEMENTS}

We thank Norma C and Albert I Geller for their constant support of the Gynecological Cancer Translation Research Program at the Case Comprehensive Cancer Center. This work was supported by grants from The Mary Kay Foundation (AD \& RX), the Eunice Kennedy Shriver National Institute Of Child Health \& Human Development of the National Institutes of Health under the NIH Director's New Innovator Award number DP2HD084068 (RX, The National Cancer Institute Award number R011CA197780$01 \mathrm{~A} 1$ (AD)) and The Young Scientist Foundation (AD). This research was supported by the Athymic Animal and Xenograft Core Facility and the Cytometry \& Imaging Microscopy Core Facility of the Case Comprehensive Cancer Center (P30CA043703).

\section{REFERENCES}

1 Siegel R, Ma J, Zou Z, Jemal A. Cancer statistics, 2014. CA Cancer J Clin 2014; 64: 9-29.

2 Cooke SL, Brenton JD. Evolution of platinum resistance in high-grade serous ovarian cancer. Lancet Oncol 2011; 12: 1169-1174.

3 Swinney DC, Anthony J. How were new medicines discovered? Nat Rev Drug Discov 2011; 10: 507-519.

4 Scannell JW, Blanckley A, Boldon H, Warrington B. Diagnosing the decline in pharmaceutical R\&D efficiency. Nat Rev Drug Discov 2012; 11: 191-200.

5 Hurle MR, Yang L, Xie Q, Rajpal DK, Sanseau P, Agarwal P. Computational drug repositioning: from data to therapeutics. Clin Pharmacol Ther 2013; 93: 335-341.

6 Ashburn TT, Thor KB. Drug repositioning: identifying and developing new uses for existing drugs. Nat Rev Drug Discov 2004; 3: 673-683.

$7 \mathrm{Xu} \mathrm{R}$, Wang Q. PhenoPredict: a disease phenome-wide drug repositioning approach towards schizophrenia drug discovery. J Biomed Inform 2015; 56: 348-355.

8 Chen Y, Xu R. Context-sensitive network-based disease genetics prediction and its implications in drug discovery. Bioinformatics 2017; 33: 1031-1039.

9 Chen Y, Xu R. Phenome-based gene discovery provides information about Parkinson's disease drug targets. BMC Genomics 2016; 17: 493.

10 Chen Y, Cai X, Xu R. Combining human disease genetics and mouse model phenotypes towards drug repositioning for parkinson's disease. AMIA Annu Symp Proc 2015; 2015: 1851-1860.

11 Wang Q, Xu R. DenguePredict: an integrated drug repositioning approach towards drug discovery for dengue. AMIA Annu Symp Proc 2015; 2015: $1279-1288$.

12 Chen Y, Xu R. Network-based gene prediction for Plasmodium falciparum malaria towards genetics-based drug discovery. BMC Genomics 2015; 16: S9.

13 Chen Y, Xu R. Drug repurposing for glioblastoma based on molecular subtypes. J Biomed Inform 2016; 64: 131-138.
14 Chen Y, Gao Z, Wang B, Xu R. Towards precision medicine-based therapies for glioblastoma: interrogating human disease genomics and mouse phenotypes. BMC Genomics 2016; 17: 516.

15 Chen Y, Li L, Zhang G-Q, Xu R. Phenome-driven disease genetics prediction toward drug discovery. Bioinformatics 2015; 31: i276-i283.

16 Cai X, Chen Y, Gao Z, Xu R. Explore small molecule-induced genome-wide transcriptional profiles for novel inflammatory bowel disease drug. AMIA Jt Summits Transl Sci Pro 2016; 2016: 22-31.

17 Xu R, Wang Q. A genomics-based systems approach towards drug repositioningfor rheumatoid arthritis. BMC Genomics 2016; 17: 518.

18 Brasky TM, Liu J, White E, Peters U, Potter JD, Walter RB et al. Non-steroidal antiinflammatory drugs and cancer risk in women: results from the Women's Health Initiative. Int J Cancer 2014; 135: 1869-1883.

19 Murphy MA, Trabert B, Yang HP, Park Y, Brinton LA, Hartge P et al. Non- steroidal anti-inflammatory drug use and ovarian cancer risk: findings from the NIH-AARP Diet and Health Study and systematic review. Cancer Causes Control 2012; 23: 1839-1852.

20 Trabert B, Ness RB, Lo-Ciganic W-H, Murphy MA, Goode EL, Poole EM et al. Aspirin, nonaspirin nonsteroidal anti-inflammatory drug, and acetaminophen use and risk of invasive epithelial ovarian cancer: a pooled analysis in the Ovarian Cancer Association Consortium. J Natl Cancer Inst 2014; 106: djt431.

21 Baandrup L, Faber MT, Christensen J, Jensen A, Andersen KK, Friis S et al. Nonsteroidal anti-inflammatory drugs and risk of ovarian cancer: systematic review and meta-analysis of observational studies. Acta Obstet Gynecol Scand 2013; 92: 245-255.

22 Li J, Zheng S, Chen B, Butte AJ, Swamidass SJ, Lu Z. A survey of current trends in computational drug repositioning. Brief Bioinform 2016; 17: 2-12.

23 Dudley JT, Deshpande T, Butte AJ. Exploiting drug-disease relationships for computational drug repositioning. Brief Bioinform 2011; 12: 303-311.

24 Dudley JT, Sirota M, Shenoy M, Pai RK, Roedder S, Chiang AP et al. Computational repositioning of the anticonvulsant topiramate for inflammatory bowel disease. Sci Transl Med 2011; 3: 96ra76.

25 Sirota M, Dudley JT, Kim J, Chiang AP, Morgan AA, Sweet-Cordero A et al. Discoveryand preclinical validation of drug indications using compendia of public gene expression data. Sci Transl Med 2011; 3: 96ra77.

26 Bult CJ, Eppig JT, Kadin JA, Richardson JE, Blake JA. Mouse Genome Database Group, The Mouse Genome Database (MGD): mouse biology and model systems. Nucleic Acids Res 2007; 36: D724-D728.

27 Hoehndorf R, Hiebert T, Hardy NW, Schofield PN, Gkoutos GV, Dumontier M Mouse model phenotypes provide information about human drug targets. Bioinformatics 2014; 30: 719-725.

28 McGettigan P, Roderick P, Mahajan R, Kadam A, Pollock AM. Use of fixed dose combination (FDC) drugs in India:central regulatory approval and sales of fdcs containing non-steroidal anti-inflammatory drugs (NSAIDs), metformin, or psychotropic drugs. PLOS Med 2015; 12: e1001826.

29 Liu R, Zheng J, Li C, Pang Y, Zheng Q, Xu X et al. Celecoxib induces epithelialmesenchymal transition in epithelial ovarian cancer cells via regulating ZEB1 expression. Arch Gynecol Obstet 2015; 291: 1361-1369.

30 Schmidt M, Christiansen CF, Horváth-Puhó E, Glynn RJ, Rothman KJ, Sørensen HT. Non-steroidal anti-inflammatory drug use and risk of venous thromboembolism. J Thromb Haemost 2011; 9: 1326-1333.

31 Ungprasert $\mathrm{P}$, Srivali N, Wijarnpreecha K, Charoenpong P, Knight EL. Non- steroidal anti-inflammatory drugs and risk of venous thromboembolism: a systematic review and meta-analysis. Rheumatology 2015; 54: 736-742.

32 Moore RA, Adel N, Riedel E, Bhutani M, Feldman DR, Tabbara NE et al. High incidence of thromboembolic events in patients treated with cisplatin-based chemotherapy: a large retrospective analysis. J Clin Oncol 2011; 29: 3466-3473.

33 Saadeh FA, Norris L, O'Toole S, Gleeson N. Venous thromboembolism in ovarian cancer: incidence, risk factors and impact on survival. Eur J Obstet Gynecol Reprod Biol 2013; 170: 214-218.

34 Wang C-J, Wang J-W, Weng L-H, Hsu C-C, Huang C-C, Yu P-C. Prevention of deepvein thrombosis after total knee arthroplasty in Asian patients. Comparison of low-molecular-weight heparin and indomethacin. J Bone Joint Surg Am 2004; 86-A: 136-140.

35 Heidel FH, Bullinger L, Feng Z, Wang Z, Neff TA, Stein L et al. Genetic and pharmacologic inhibition of ??-catenin targets imatinib-resistant leukemia stem cells in CML. Cell Stem Cell 2012; 10: 412-424.

36 Nagaraj AB, Joseph P, Kovalenko O, Singh S, Armstrong A, Redline R et al. Critical role of $W n t / \beta$-catenin signaling in driving epithelial ovarian cancer platinum resistance. Oncotarget 2015; 6: 23720-23734.

37 Gurpinar E, Grizzle WE, Piazza GA. NSAIDs inhibit tumorigenesis, but how? Clin Cancer Res 2014; 20: 1104-1113.

38 Kino $Y$, Kojima F, Kiguchi K, Igarashi R, Ishizuka B, Kawai S. Prostaglandin E2 production in ovarian cancer cell lines is regulated by cyclooxygenase-1, not cyclooxygenase-2. Prostaglandins Leukot Essent Fatty Acids 2005; 73: 103-111. 
Using DrugPredict to rapidly identify potent drug candidates

AB Nagaraj et al

414

39 Nakanishi M, Rosenberg DW. Multifaceted roles of PGE2 in inflammation and cancer. Serin Immunopathol 2013; 35: 123-137.

40 Ahnen DJ. Colon cancer prevention by NSAIDs: what is the mechanism of action? Eur J Surg Suppl 1998. 111-114.

41 Ulrich CM, Bugler J, Potter JD. Non-steroidal anti-inflammatory drugs for cancer prevention: promise, perils and pharmacogenetics. Nat Rev Cancer 2006; 6: $130-140$.

42 Goessling W, North TE, Loewer S, Lord AM, Lee S, Stoick-Cooper CL et al. Genetic interaction of PGE2 and Wit signaling regulates developmental specification of stem cells and regeneration. Cell 2009; 136: 1136-1147.

43 Flesken-Nikitin A, Huang C-I, Chen C-Y, Michurina TV, Enikolopov G, Nikitin AY. Ovarian surface epithelium at the junction area contains a cancer-prone stem cell niche. Nature 2013; 495: 241-245.

44 Laden CN, Goodman B, Kate AA, Steg AD, Nick AM, Stone RL et al. Targeting aldehyde dehydrogenase cancer stem cells in ovarian cancer. Mol Cancer Sher 2010; 9: 3186-3199.

45 Condello S, Morgan CA, Nagdas S, Gao L, Turk J, Hurley TD et al. $\beta$-Cateninregulated ALDH1A1 is a target in ovarian cancer spheroids. Oncogene 2015; 34: 2297-2308.

46 Vole BL, D'Souza T, Becker KG, Wood WH, Chang Y, Wersto RP et al. Non- steroidal anti-inflammatory drugs decrease E2F1 expression and inhibit cell growth in ovarian cancer cells. PLUS One 2013; 8: e61836.
47 Ye F, Ching Y, Breschi A, Vierstra J, Wu W, Ryba T et al. A comparative encyclopedia of DNA elements in the mouse genome. Nature 2014; 515: 355-364.

48 Bell D, Berchuck A, Birrer M, Chen J, Crater DW, Do F et al. Integratedgenomic analyses of ovarian carcinoma. Nature 2011; 474: 609-615.

49 Davis AP, King BL, Mockus S, Murphy CG, Saraceni-Richards C, Rosenstein M et al. The comparative toxicogenomics database: update 2011. Nucleic Acids Res 2011; 39: D1067-D1072.

50 Wishart DS, Knox C, Gro AC, Shrivastava S, Hassanali M, Stothard P et al. DrugBank: a comprehensive resource for in silico drug discovery and exploration. Nucleic Acids Res 2006; 34: D668-D672.

This work is licensed under a Creative Commons AttributionNonCommercial-NoDerivs 4.0 International License. The images or other third party material in this article are included in the article's Creative Commons license, unless indicated otherwise in the credit line; if the material is not included under the Creative Commons license, users will need to obtain permission from the license holder to reproduce the material. To view a copy of this license, visit http:// creativecommons.org/licenses/by-nc-nd/4.0/

(c) The Author(s) 2018

Supplementary Information accompanies this paper on the Oncogene website (http://www.nature.com/onc)

Oncogene (2018) 403-414 